\title{
tic\&société
}

Vol. 13, $\mathbf{N}^{\circ}$ 1-2 | 1er semestre 2019 - 2ème semestre 2019

Les industries culturelles à la conquête des

plateformes?

\section{Données personnelles et plateformes numériques : sophistication et concentration du marché publicitaire}

Thomas GUIGNARD

\section{OpenEdition}

\section{Journals}

Édition électronique

URL : http://journals.openedition.org/ticetsociete/3377

DOI : 10.4000/ticetsociete.3377

Éditeur

Association ARTIC

Édition imprimée

Pagination : 43-69

\section{Référence électronique}

Thomas GUIGNARD, « Données personnelles et plateformes numériques : sophistication et

concentration du marché publicitaire », tic\&société [En ligne], Vol. 13, № 1-2। 1er semestre 2019 -

2ème semestre 2019, mis en ligne le 20 avril 2019, consulté le 14 mai 2019. URL : http://

journals.openedition.org/ticetsociete/3377 ; DOI : 10.4000/ticetsociete.3377

Ce document a été généré automatiquement le 14 mai 2019

Licence Creative Commons 


\title{
Données personnelles et plateformes numériques : sophistication et concentration du marché publicitaire
}

\author{
Thomas GUIGNARD
}

1 Si les plateformes numériques constituent un enjeu de premier ordre sur les plans culturel et économique (Bouquillion, Miège, Mœglin, 2013), elles ont révélé une nouvelle « ressource essentielle » dans le paysage numérique mondial : les algorithmes et les data. À très grande vitesse, un nombre grandissant de domaines - la culture, le sport et l'information, mais aussi la médecine, les transports, les territoires, ou encore la finance sont désormais outillés par des algorithmes de plus en plus performants (Cardon, 2015). Ils organisent et structurent les informations, aident à prendre des décisions ou automatisent notre quotidien. Afin de justifier le développement de ces outils prédictifs, les promoteurs des Big Data disqualifient la pertinence des intermédiaires traditionnels non automatisés.

Sur le marché publicitaire, les acteurs du secteur et notamment les médias « traditionnels 1 " sont confrontés à des mutations profondes portées par de nouveaux dispositifs d'intermédiation dont les performances sont étroitement liées à la maîtrise des data. Il apparaît ainsi que ces acteurs, américains pour les plus influents (Google et Facebook notamment), occupent une fonction centrale sur le marché de la publicité numérique en maîtrisant notamment la collecte et la gestion des données personnelles permettant d'optimiser la relation client et l'appariement entre annonceurs et consommateurs. L'activité de ces nouveaux services repose ainsi sur leur capacité à recueillir des données et à les exploiter pour rendre le système plus efficace. Plus le nombre de participants se développe, plus le volume d'informations à leur sujet augmente. Ainsi, mécaniquement, plus le service est attractif plus il renforcera son efficacité, un phénomène appelé datadriven network effects (Parker, Van Alstyne et Choudary, 2016). 
3 La montée en puissance de la collecte des données personnelles a lieu dans une économie globalisée et dérégulée qui favorise la concentration extrême de ressources autour d'une poignée d'acteurs expliquant en partie la domination d'un duopole formé par Google et Facebook sur le marché publicitaire numérique mondial. Aussi ces plateformes publicitaires intégrées semblent-elles «disqualifier » les acteurs "traditionnels» du marché de la publicité. Tout d'abord, contrairement à la publicité «classique», ces dispositifs publicitaires autorisent un degré de ciblage et de personnalisation beaucoup plus élevé tout en permettant aux annonceurs de configurer leurs campagnes publicitaires par l'intermédiaire de modules automatisés et une panoplie de paramétrages inédits (temporalité, centre d'intérêt, budget alloué par jour, localisation géographique, etc.).

4 Les serveurs d'annonces de ces plateformes enregistrent chaque action en temps réel permettant aux annonceurs d'optimiser leurs insertions publicitaires et de suivre leurs campagnes : ils peuvent, par exemple, connaître le profil précis (âge, sexe, niveau de scolarité, etc.) et la localisation géographique des personnes cliquant sur la publicité grâce à des logiciels ou des applications dédiés mis à disposition par le gestionnaire de plateformes. Aussi, leur capacité à recueillir des données au fil du temps et à les utiliser pour rendre le système plus efficace a permis aux plateformes publicitaires de Google et de Facebook de croître très rapidement, jusqu'à dominer démesurément le marché publicitaire numérique mondial : en 2017, les plateformes publicitaires des deux acteurs concentrent en effet plus de $70 \%$ des recettes publicitaires sur mobile à l'échelle mondiale et plus de $90 \%$ de la croissance annuelle (2016-2017) du marché de la publicité numérique aux États-Unis et en France (Rodger et Thorson, 2017).

5 Cette hégémonie inédite met-elle en péril les régies publicitaires locales/nationales ainsi que l'équilibre économique du paysage médiatique reposant en grande partie sur les recettes publicitaires? Quels sont les modes de fonctionnement et de déploiement de ces nouveaux services? Quel est leur poids sur le marché publicitaire et comment s'y marquent leurs interventions? De quelles natures sont les rapports avec les annonceurs? C'est là autant de questions qu'il semble important de traiter pour tenter de comprendre l'évolution du marché publicitaire et de l'économie des médias.

6 Le présent article a pour objectif d'analyser l'évolution de l'écosystème publicitaire et médiatique, ses principales transformations et répercussions socioéconomiques et, notamment, l'influence de nouveaux intermédiaires numériques sur le marché de la publicité et sur le paysage médiatique. Une étude des usagers s'avérerait intéressante à ce propos. Néanmoins, nous avons choisi de nous focaliser sur le niveau macro des acteurs à travers une approche socioéconomique des médias et de la publicité.

7 L'économie des médias apparait comme un champ de recherche opératoire pour mieux comprendre et cerner les mutations à l'œuvre. Les conséquences de l'interaction stratégique entre deux marchés, le marché des médias et le marché de la publicité (Sonnac, 2013) sont au cœur de notre analyse. L'économie industrielle nous aidera à mieux comprendre le fonctionnement des médias et des plateformes publicitaires. Notre approche emprunte ainsi pour l'essentiel aux cadres théoriques de la sociologie et de l'économie adaptées à une perspective en sciences de l'information et de la communication. La sociologie économique des médias s'avèrera particulièrement pertinente pour étudier les transactions entre annonceurs et journaux, radios ou télévisions et pour analyser l'évolution des relations entre les nouveaux intermédiaires de la publicité en ligne, les annonceurs et les médias traditionnels. 
8 D'un point de vue méthodologique, nous nous appuierons sur des données de seconde main relatives au marché publicitaire issues d'études existantes, tout en inscrivant nos analyses dans une perspective critique de la socioéconomie des médias inhérente à notre champ disciplinaire, les sciences de l'information et de la communication, afin de redonner aux biens informationnels une dimension symbolique souvent éludée dans des approches économétriques. Nous faisons ainsi l'hypothèse que le numérique est susceptible de modifier l'équilibre des filières des industries culturelles et médiatiques. La présente étude sur l'influence et la domination de puissantes plateformes publicitaires rendant plus incertaine la possibilité de financer la production de contenus informationnels s'insère dans cette perspective.

\section{Les data au cœur de l'architecture et du fonctionnement des plateformes publicitaires numériques}

9 L'analyse de Google et de Facebook au prisme de l'économie industrielle révèle l'existence de plusieurs niveaux de plateformes traduisant le caractère multiversant de leurs activités. L'architecture des services de ceux-ci et les effets de réseaux inhérents permettent de les rattacher au modèle de la plateforme. La partie publicitaire de leur activité mérite également une même appellation. Même si elle ne peut être dissociée des autres activités de ces groupes internationaux, leur mode de fonctionnement repose également sur les principaux traits structurants d'une plateforme : la mise en relation de plusieurs versants, la présence d'effet de réseaux importants tout comme la sophistication (traduisant les actions d'amélioration et de perfectionnement d'un objet ou d'un dispositif grâce à des techniques avancées) et l'industrialisation des insertions publicitaires témoignent de cette tendance. Les plateformes fournissent ainsi une infrastructure ouverte pour ces interactions et définissent les conditions et la gestion des relations entre les deux versants (Brousseau et Curien, 2001). Ces plateformes numériques, animées par la technologie et des outils logiciels sophistiqués, qui relient de plus en plus finement et rapidement plusieurs catégories d'acteurs (généralement deux versants, les annonceurs et les éditeurs pour les plateformes liées à la publicité numérique), permettent dans bien des domaines de surclasser les modèles d'organisation traditionnels. Dans la littérature économique, la plateforme est souvent opposée au modèle marchand et son fonctionnement linéaire (Hagiu, 2007). Ainsi, bien que le marché de l'intermédiation ne soit pas un phénomène nouveau, l'économie numérique a permis une généralisation du modèle à de nombreux pans de l'économie. Le dispositif permet alors de relier deux versants par un système d'appariement qui est au cœur de la création de valeur d'une plateforme. Compte tenu de sa modularité et de son ouverture sur l'extérieur, une plateforme compétitive dépend largement de la valeur créée par des acteurs tiers. C'est précisément en cela que la plateforme est avant tout un agent d'intermédiation (au sens de mise en relation des acteurs) : elle remplit ainsi certaines fonctions économiques qui sont à la fois informationnelles (outil de recherche et prescription) et transactionnelles (sécurisation des paiements, gestion logistique, etc.) (Bullich et Guignard, 2011).

10 Dans ce processus, les données constituent une dimension fondamentale du modèle : plus la plateforme travaille son système grâce à des algorithmes pour collecter, organiser, 
trier, analyser et interpréter les données, plus précis seront les filtres, plus pertinentes et utiles seront les informations échangées, et ainsi plus efficace sera l'appariement entre les différents versants. Ces plateformes doivent ainsi avoir une stratégie explicite d'acquisition de données (Parker, Van Alstyne et Choudary, 2016).

Le caractère multiversant (two sided ou multi-sided market) de ces dispositifs publicitaires renvoie ainsi à un autre élément constitutif du modèle de la plateforme: les effets de réseaux et la captation des externalités de réseaux par le gestionnaire de plateforme. Bien que devenue récemment prégnante dans les filières liées aux TIC, cette caractéristique n'est absolument pas nouvelle, ni pour le secteur publicitaire - une telle organisation est en effet consubstantielle du modèle médiatique de flot ou de celui de la presse, par exemple (Sonnac, 2006) - ni pour d'autres secteurs économiques, comme le minitel, les systèmes de cartes bancaires, les affiliations à des enseignes commerciales, etc. (Gawer, 2009 ; Rochet et Tirolle, 2003)

12 Le caractère distribué des annonceurs utilisant les plateformes publicitaires de Google et de Facebook apparaît comme élément structurant de leur réussite : leur stratégie ne vise pas, contrairement à de nombreuses autres stratégies industrielles, à intégrer, au sein d'une même entité, les différentes fonctions et activités d'une filière donnée, mais, au contraire, cherche à favoriser la modularité et la pluralité des acteurs associés. Aussi, avec ces plateformes, la nature de l'offre publicitaire change. Elle est dorénavant déverrouillée, la capacité inutilisée (spare capacity) est exploitée. Alors que les médias ont fonctionné sur une offre (un inventaire) structurée, ces nouvelles plateformes fonctionnent sur une offre externalisée. Comme nous l'avons expliqué, la valeur intrinsèque des plateformes de Google et de Facebook se trouve principalement dans les effets de réseaux qu'elle crée. Ceux-ci, en créant des boucles rétroactives d'autorenforcement (self-reinforcing feedback loops), grossissent leur base d'utilisateurs souvent avec un minimum d'effort ou d'investissement pour le gestionnaire de la plateforme (notamment pour les leaders, winner-take-all markets2).

13 L'efficacité d'une plateforme publicitaire repose en partie sur sa capacité à attirer des annonceurs et des utilisateurs très éclatés géographiquement. Cette caractéristique permet aux plateformes d'attirer aussi bien des annonceurs ayant un ancrage local que des entreprises multinationales. Les effets de réseaux, pour les plateformes les plus compétitives, s'inscrivent ainsi dans une dimension internationale: c'est pourquoi les régies publicitaires classiques essentiellement structurées à partir des territoires nationaux ne peuvent souvent rivaliser avec la puissance locale et internationale des plateformes comme Facebook et Google. Ce point explique également l'intensification de la concentration dans le secteur de la publicité numérique.

\section{Les data et les algorithmes au cœur de la reconfiguration du marché publicitaire}

14 S'interroger sur les relations économiques actuelles entre médias et publicité nécessite de connaître la relation historique de ce couple. La publicité est un intermédiaire entre l'offre et la demande qui sert à améliorer le fonctionnement du marché. La publicité, comme la presse, est née dans le cadre du développement capitaliste, lorsque l'économie de pénurie qui favorise l'offre cède la place à une économie où la demande est le principal acteur; elle demeure liée à ce système (Eveno, 2004). 
15 La part de la publicité dans l'économie des médias a toujours fluctué au cours de l'histoire. Dans la grande presse de la fin du $19^{\mathrm{e}}$ siècle, les recettes publicitaires sont cantonnées à $20 \%$ des recettes totales en France. Il faut en effet attendre les années 1950 et l'entrée de la France dans l'âge publicitaire pour qu'une partie importante des recettes de la presse provienne de la publicité et des annonces. De 1947 à 1990, les recettes publicitaires du quotidien Le Monde représentent au minimum $40 \%$ des recettes totales et atteignent même $59 \%$ en 1970. À cette même période, d'autres journaux apparaissent encore plus dépendants des investissements publicitaires des annonceurs: ceux-ci constituent $85 \%$ des recettes totales du Figaro et du quotidien économique Les Échos. Ils sont en revanche moins importants dans la presse quotidienne régionale, puisqu'ils génèrent la moitié des recettes du secteur (Eveno, 2004).

16 Aujourd'hui, les médias dépendent encore des recettes publicitaires même si elles ont perdu du poids ces dernières années. En 2017, la part de la publicité dans l'économie de la presse française avoisinerait les 30 \% (hors petites annonces ${ }^{3}$ ). En revanche, les chaînes de radio et de télévision privées dépendent intégralement des recettes publicitaires, hormis les chaînes de télévision payantes comme Canal + et certaines chaînes du câble et du satellite $^{4}$ qui ont un modèle mixte mêlant frais d'abonnement et recettes publicitaires. Aussi les médias traditionnels dans leur ensemble dépendent-ils encore largement des revenus publicitaires (Toussaint-Desmoulins, 2015).

Les dépenses "médias » sont réparties en cinq catégories : la télévision, la presse, la radio, la publicité extérieure et le cinéma. Depuis les années 2000, Internet est considéré comme le sixième support de communication comptabilisé dans les dépenses médias. Dans les années 2000, la part des recettes publicitaires d'Internet était nulle; depuis, elle n'a cessé de croître, pour atteindre $35 \%$ en 2017. Sur la même période, la part des supports historiques a décru de façon continue, passant de $100 \%$ à $65 \%$ de la totalité des dépenses médias sur la même période.

La numérisation de l'information s'accompagne d'une explosion de contenus qui a engendré une fragmentation accrue des usages (Evans et Schmalensee, 2016). Cette tendance forte donne à l'appariement une place névralgique. La fonction d'intermédiation reconfigure ainsi les équilibres et les positions sur le marché de la publicitaire aujourd'hui dominé dans un nombre croissant de pays par la publicité numérique (comme en France, où, en 2017, les investissements liés à la publicité numérique ont dépassé ceux de télévision ${ }^{5}$ ).

Il semble important de revenir sur l'évolution de la publicité numérique afin de mieux comprendre et cerner la situation actuelle. Depuis les années 1990, la publicité numérique repose sur deux leviers principaux, à savoir le Search Engine Advertising (SEA ou search), lié aux moteurs de recherche par l'achat de mots-clés (le programme AdWords de Google domine par exemple largement le marché français), et le display, qui désigne les formes de publicité numérique utilisant des éléments graphiques ou vidéos.

Dans le cas du display, les ciblages par emplacements (à travers le choix, par l'annonceur, de sites ou de pages web susceptibles d'attirer des utilisateurs sensibles à son offre), contextuels (à travers le choix, par l'annonceur, de mots-clés et de thèmes liés à ses produits ou ses offres) ou sociodémographiques ont longtemps été les seules modalités mises à disposition des annonceurs. La complexification des parcours d'achat et le besoin toujours croissant d'un ciblage plus pertinent ont progressivement accordé aux données personnelles une importance singulière. L'évolution de la publicité dite « display » illustre 
bien la montée des données au sein de la publicité numérique à partir des années 2000, avec une intensification particulièrement soutenue au cours des années 2010. On peut distinguer trois phases principales dans les pratiques d'achat display (Allary et Balusseau, 2018) :

- À partir du milieu des années 1990, les achats de gré à gré display dit «classiques» apparaissent. Dans cette configuration, un site éditorial (comme Le Monde) négocie directement avec un annonceur (comme Sony Music) pour commercialiser son inventaire à un prix fixe négocié (le plus souvent au coût par mille [CPM], soit le coût de mille bannières affichées);

- À partir du début des années 2000, des acteurs appelés «Ad Networks » apparaissent. Ceux-ci agrègent les inventaires invendus et permettent un ciblage contextuel (les Ad Networks étant souvent spécialisés par cible ou par thème, comme "cadres urbains", "seniors » ou «amateurs de produits high-tech»). Ces acteurs sont nés pour répondre à la difficulté des éditeurs de contenus de commercialiser les millions d'impressions disponibles et invendus sur leurs sites;

- À partir des années 2010, les Ad Exchanges prennent position sur le marché. Ces nouvelles places de marché permettent de connecter les éditeurs (leurs inventaires) et les annonceurs qui enchérissent en temps réel pour y insérer leurs messages publicitaires. Le prisme évolue par rapport aux deux modes précédents : dans le ciblage et la fixation du prix, on s'intéresse désormais autant à l'utilisateur qu'à l'emplacement de la diffusion du message publicitaire. Ces achats aux enchères constituent ce que l'on appelle le Real Time Bidding (ou RTB), qui représente plus de $80 \%$ des achats en programmatique ${ }^{6}$ en 2017 (Allary et Balusseau, 2018).

21 Aussi, ces nouveaux acteurs dans le champ publicitaire, liant l'informatique et l'intermédiation, proposent-ils un appariement entre un inventaire (d'espaces publicitaires) proposé pour des éditeurs (fournisseurs de contenus ou services) et des annonceurs. Le fonctionnement des places de marché publicitaires nommées Ad Exchange Platform illustre ainsi bien la sophistication et l'industrialisation du marché publicitaire numérique. L'objectif est de relier deux versants, les éditeurs (SSP - Supply Side Platform) et les annonceurs (DSP - Demand Side Platform) et de toucher ainsi une audience très ciblée (Real Time Audience) via un système de tarification basé sur des enchères en temps réel ( Real-Time Bidding) (Guibert, Rebillard et Rochelandet, 2016).

Les opérateurs de ces plateformes (SSP ou DSP) peuvent être indépendants (on en compte une quinzaine qui sont actifs en France) alors que d'autres sociétés, comme Google (Double Click) et Facebook (Atlas), sont à la fois des SSP et des DSP. Ces dernières proposent ainsi un Ad Exchange complet (Lallement, et Serris et Varenne, 2014). Acheter sur les plateformes d'Ad Exchange est un nouveau métier qui nécessite une infrastructure technique permettant de surveiller le flux des impressions, d'évaluer celles-ci, de choisir une enchère, puis de diffuser une annonce.

Ces plateformes publicitaires autorisent ainsi des transactions qui étaient impossibles auparavant tant pour des raisons pratiques que budgétaires. Le programme AdSense lancé par Google permet ainsi à une kyrielle de producteurs de contenus (sites internet, blogs, forums...) de proposer des espaces publicitaires sur tout un pan du Web pour lequel des gestionnaires de sites ou d'applications n'avaient pas les moyens humains et financiers de gérer une régie publicitaire en interne. L'appariement automatisé proposé par AdSense entre une multitude d'annonceurs (petits ou gros) et une myriade de sites (souvent avec une audience limitée) a entraîné un abaissement important des coûts de la rencontre entre offre et demande publicitaire. Ce type de marché conduit structurellement à des 
marchés très concentrés, car les externalités et les effets de réseaux évoqués précédemment favorisent largement les acteurs les plus puissants capables de gérer un inventaire et une base d'utilisateurs très importants (Smyrnaios, 2017).

L'innovation liée au ciblage comportemental participe ainsi à transformer la logique de fonctionnement de l'activité publicitaire (Ouakrat, 2012). Alors que la publicité traditionnelle peut atteindre un très grand nombre de personnes dont certaines sont dans le jargon de la publicité des "audiences inutiles» (wasted exposures), les serveurs publicitaires ont le potentiel de diriger des messages spécifiques vers des personnes spécifiques. Les achats programmatiques s'inscrivent ainsi dans cette tendance et engendrent des bouleversements profonds reconfigurant l'industrie de la publicité et des médias. Inexistants avant 2012, les achats programmatiques représentaient en 2017 environ $60 \%$ des achats sur le marché français de la publicité numérique ${ }^{7}$.

Il y a quelques années lorsqu'on voulait acheter des insertions publicitaires sur divers médias, les agences et les annonceurs devaient négocier avec des commerciaux. La lourdeur administrative et le manque d'efficacité publicitaire (en raison notamment d'un manque de critères de ciblage) ont amené la profession à s'orienter vers l'achat programmatique. Ce dernier caractérise ainsi des achats réalisés par l'intermédiaire d'un logiciel qui automatise les processus des transactions: sélection des emplacements publicitaires, définition des prix... Ils sont donc associés au fait d'utiliser un algorithme pour réaliser l'achat de son espace publicitaire, en opposition au traditionnel processus impliquant des négociations, des allers-retours de plans média, et des ordres d'insertion manuels.

En automatisant le processus d'achat des inventaires numériques, certains grands médias comme le New York Times ont ainsi pu automatiser le processus d'achat média et rationaliser leur force de vente. En 2015, le journal américain a par exemple décidé que tous les achats de bandeaux simples sur ses sites et ses applications seraient dorénavant possibles uniquement en mode programmatique et qu'il mobiliserait son équipe de ventes réduite sur les grands comptes (Renaud, 2017). Les éditeurs distinguent généralement l'espace publicitaire de premier choix (ou premium) des autres. La commercialisation des espaces dits « premium » reste généralement internalisée, tandis que les espaces de second rang sont vendus à partir de plateformes spécialisées (Ad Exchange). On notera cependant que cette distinction tend à s'estomper, comme en témoigne la montée significative de la publicité programmatique sur l'ensemble du marché.

Le " goulot d'étranglement » lié à la rareté des espaces publicitaires sur les grands médias s'est ainsi desserré grâce à ces plateformes, qui ont considérablement baissé les coûts logistiques liés à la diffusion, à la distribution et à l'appariement des espaces publicitaires. Par ailleurs, la nature même du marché programmatique nécessite une connaissance fine des utilisateurs et son efficacité ne croît qu'en collectant et qu'en traitant un nombre croissant de données. Avec la sophistication des régies publicitaires numériques, on change ainsi de paradigme, passant d'un espace publicitaire valorisé pour le contenu dans lequel il s'insère à une valorisation basée sur la pertinence du profil qui va consulter ce contenu. Une nouvelle configuration s'installe, dans laquelle l'exploitation à grande échelle des données oriente les éditeurs de contenus vers un rôle de marchandisation des données comportementales de son audience (Ouakrat, 2012). 


\section{Facebook et Google, une position dominante sur le marché de la publicité numérique}

créés hors du champ médiatique et s'inscrivent dans le domaine de l'ingénierie technique (Allary et Balusseau, 2018). L'efficacité liée à la maîtrise des data a amené les médias traditionnels à collaborer avec ces intermédiaires d'un nouveau genre. De nombreux spécialistes de la publicité numérique estiment que le modèle des Ad Exchanges va se généraliser à l'ensemble de la publicité en ligne, voire à certains médias traditionnels (radio, télévision) dans leur version numérique. Ils anticipent également une poursuite de la concentration du trafic autour des acteurs les plus importants, parmi lesquels on trouve Double Click (Google), AppNexus (plateforme indépendante, qui est néanmoins détenue à $20 \%$ par Microsoft) et Atlas (Facebook) (Lallement, Serris et Varenne 2014).

En comparaison avec les principaux concurrents, Google et Facebook disposent de moyens financiers colossaux leur conférant un pouvoir de marché disproportionné par rapport aux acteurs "traditionnels » essentiellement structurés autour de territoires nationaux. Ce pouvoir leur permet d'ériger des barrières à l'entrée très élevées sur le marché de la publicité numérique en investissant massivement en $\mathrm{R}$ et $\mathrm{D}^{8}$ et en infrastructures, leur garantissant une avance technologique (Smyrnaios, 2017). Les manœuvres du duopole basées sur le rachat en nombre des concurrents et des startups à fort potentiel de croissance en sont une autre illustration. Google et Facebook ont ainsi su profiter de leur avance dans ce domaine pour s'affranchir des intermédiaires du secteur en retirant leur offre des plateformes Ad Exchange concurrentes. En acquérant la société Doubleclick en 2007, Google avait ainsi bien anticipé cette orientation du marché vers la publicité programmatique.

Aussi, pour acheter des impressions sur le réseau display de Google, un annonceur n'a-t-il pas d'autre choix que de passer par deux DSP de Google: AdWords ou Doubleclick Bid Manager (DBM). Chaque Ad Exchange permet d'accéder à des inventaires différents : DoubleClick Ad Exchange de Google permet d'accéder à l'inventaire display de Google (Google Display Network, AdSense et YouTube).

Le programme AdWords peut en outre être rattaché à la publicité programmatique, car il se base également sur un achat automatisé. En revanche, AdWords se limite à un éditeur spécifique et une campagne d'achat de mots-clés ne peut pas s'intégrer dans un achat programmatique multicanal. Dans le cas du search, le programme AdWords peut être considéré comme la DSP propriétaire du moteur de recherche Google permettant à un annonceur de placer ses annonces en haut de la page de résultats de Google. D'autres plateformes, comme Doubleclick Search (appartenant également à Google), permettent de se positionner sur les moteurs Bing, Baidu et Yahoo, en plus de Google.

Dans le domaine de la traçabilité de ses utilisateurs, Google fait figure de "référence ", notamment grâce à ses nombreux services, gratuits pour la plupart, qui permettent à l'internaute de gérer toutes ses activités numériques (recherches, actualités, musique, vidéos, messagerie par un compte utilisateur unique). Cette panoplie de services permet à Google de proposer des solutions publicitaires de plus en plus fines et complètes : depuis janvier 2017, les annonceurs peuvent, par exemple, cibler leur publicité sur YouTube en tenant compte de l'historique de l'utilisateur sur le moteur de recherche Google.

tic\&société, Vol. 13, N 1-2 | 1er semestre 2019 - 2ème semestre 2019 

gracieuse aux critères de ciblage (et aux segments d'individus) disponibles sur le Google Display Network, comme les audiences affinitaires si les achats sont effectués sur le réseau de Google. La donnée est donc parfois gratuite, mais son utilisation reste toujours payante. Cette même logique favorise également le recours à DoubleClick, qui comporte une solution pour chaque étape de la mise en œuvre d'une campagne : l'hébergement des créations se fait à partir du Doubleclick Campaign Manager (l'Ad Server de Google); l'achat d'espace se déroule quant à lui sur DoubleClick Bid Manager, la diffusion se fait sur DoubleClick AdExchange (l'inventaire dont les publicités sont administrées par Google); et les retombées des campagnes publicitaires sur le site de l'annonceur sont mesurées par Google Analytics (l'outil de mesure site-centric de Google). En dehors de l'aspect financier (faible commission sur les achats par la DSP Google), les annonceurs y trouvent aussi un intérêt marketing, avec la possibilité de comprendre le parcours des internautes sur une large palette de terminaux et de plateformes grâce à l'unification des données. Il devient ainsi possible d'optimiser les campagnes et leur impact pour les différents écrans, canaux et formats, depuis la mise en ligne de la campagne jusqu'à son impact sur le site. On contrôle ainsi les paramètres médias (couverture dédupliquée des internautes entre les différents sites et plateformes, maîtrise de la répétition) (Allary et Balusseau, 2018).

le secteur de la publicité numérique, un individu est incarné par un cookie (voire un ensemble de cookies). Les seules exceptions à ce principe sont les environnements où il est obligatoire de se connecter (avec une adresse e-mail) pour utiliser le service : c'est ce qui fait la force publicitaire de Facebook et de Google, qui peuvent s'affranchir de la logique de cookies grâce aux comptes détenus par les individus sur leurs plateformes. Il s'agit ainsi d'un avantage considérable par rapport aux concurrents. C'est la raison pour laquelle Facebook a racheté l'Ad Server Atlas en 2013 : la technologie Atlas de Facebook permet de suivre un individu en utilisant un système basé sur l'identifiant Facebook, et non sur un cookie. Dans cette configuration, Facebook contraint l'utilisation de ses propres solutions de suivi pour mesurer la performance des campagnes : il n'est donc pas possible d'utiliser un Ad Server tiers dans le cas de campagnes Facebook (Allary et Balusseau, 2018). fondamental pour comprendre et cerner le succès des deux géants américains. Facebook base sa stratégie sur son dispositif Newsfeed, lequel hiérarchise l'apparition des informations dans le fil d'actualité de ses utilisateurs sur la base d'algorithmes qui prennent en considération les interactions entre les utilisateurs et la plateforme, comme les likes, les partages, les commentaires, ou encore les liens entre utilisateurs. Du côté de la production/distribution de contenus, les algorithmes prennent en considération plusieurs critères, comme le format, la popularité du fournisseur de contenus, la popularité de ses précédentes publications, etc. Le but est de fournir une architecture optimisant à la fois l'« expérience utilisateur » et les revenus liés à la publicité.

Parallèlement, de cette maturité technologique ont surgi des approches inédites de la relation au consommateur, inspirées notamment par le concept de permission marketing, popularisé par Seth Godin (2007). Le principe repose sur une relation de "confiance » entre le consommateur, qui accepte de dévoiler ses goûts et ses intérêts, et la plateforme, qui s'engage à lui proposer l'offre la plus attractive et la plus appropriée au regard de ses attentes. Facebook connaît ses utilisateurs de manière très fine grâce aux renseignements qu'ils laissent plus ou moins volontairement sur la plateforme. Les utilisateurs renseignent souvent leur profil de manière exhaustive: formation, poste occupé, âge, 
séries et marques préférées, etc., et, parallèlement, Facebook génère d'autres données liées à la localisation géographique des connexions ou encore aux actions des utilisateurs. Chaque connexion produit ainsi des données: plus l'utilisateur passe de temps et interagit avec la plateforme, plus celle-ci peut proposer des profils publicitaires ciblés à ses annonceurs.

Afin de renforcer son emprise sur le paysage publicitaire mondial, Facebook a noué d'étroites relations avec les principaux courtiers de données américains (BlueKai9, Datalogix, ou encore Acxiom). On notera que l'entreprise, en lançant ses pages «fan » en 2007, a permis aux entreprises d'entretenir une communauté. Avant 2012, le gestionnaire d'une page fan pouvait toucher l'intégralité de sa communauté gratuitement. Face au succès du service, la compagnie américaine a décidé de modifier les règles et a réussi à faire payer les gestionnaires de pages pour toucher leurs «fans». Dénommé « organic reach", ce ratio est passé progressivement de $100 \%$ à moins de $4 \%$ en 2018 . Il faut désormais passer par le programme publicitaire de Facebook pour toucher sa propre communauté.

La mise à disposition d'outils de suivi de campagnes publicitaires auprès des annonceurs apparaît comme un autre élément constitutif de la domination de Facebook et de Google sur le marché de la publicité numérique. Les annonceurs disposent en effet d'une batterie de métriques permettant d'évaluer l'efficacité d'une campagne en mesurant les actions des internautes (le taux de clic, le taux d'engagement, le taux de conversion, etc.), mais également leur profil (données sociodémographiques). La flexibilité est telle que certains annonceurs peuvent lancer plusieurs messages publicitaires et se concentrer par la suite sur celui qui enregistre les meilleurs résultats (délaissant ainsi les moins efficaces).

Ces régies publicitaires mondialisées réussissent également à attirer à la fois les petits annonceurs (comme un restaurant de quartier) et les plus grands annonceurs mondiaux, qui peuvent toucher des dizaines, voire des centaines, de millions d'utilisateurs par un interlocuteur unique et une interface unifiée pour suivre l'efficacité d'une campagne mondiale par pays. Dans cette configuration, il apparait que la valeur pour les annonceurs ne provient pas seulement du volume des utilisateurs présents sur l'autre versant, mais surtout de l'adéquation de l'audience avec leurs objectifs. Google et Facebook tendent à proposer des solutions d'achat d'espaces publicitaires de plus en plus intégrées, et il est de plus en plus difficile de distinguer DSP, Ad Exchange et SSP tant leurs solutions publicitaires sont complètes (le lancement, en 2018, du service Google Platform Marketing visant à centraliser l'ensemble des outils publicitaires de Google illustre cette orientation).

\section{Les acteurs français de la publicité numérique et le duopole Facebook/Google: dépendance et résistance}

Autre conséquence de cette reconfiguration du marché publicitaire, le rapport de force s'inverse entre annonceurs et éditeurs: dans un environnement marqué par une explosion de l'offre de contenus et des supports publicitaires, les annonceurs sont davantage en mesure de négocier les prix et d'imposer leurs exigences. La numérisation des biens informationnels s'est en effet accompagnée d'une explosion de l'offre d'espaces publicitaires, qui rend la survie des médias traditionnels bien plus complexe. La situation apparaît tenable dans un univers marqué par une concurrence limitée, mais elle devient 
plus que problématique dans un paysage où la production de contenus tout comme les supports publicitaires apparaissent illimités. De plus, il est intéressant de noter que les éditeurs, en passant par ces nouveaux intermédiaires publicitaires, sont dépossédés de la relation directe avec leur audience, renforçant ainsi leur dépendance vis-à-vis des plateformes publicitaires numériques.

41 Aussi, la croissance de ce marché de la publicité numérique n'est pas synonyme de pluralité. Le duopole formé par Google et Facebook a en effet accaparé $92 \%$ de la croissance enregistrée entre 2016 et 2017 sur le marché de la publicité numérique en France ${ }^{10}$. En France, au premier semestre 2017, les segments de la recherche en ligne ( search) et des réseaux sociaux représentaient $71 \%$ du marché de la publicité numérique. Google et Facebook, les leaders respectifs sur ces deux segments de marché, tendent ainsi à renforcer leur position dominante: ce que l'on abordait auparavant comme une tendance est devenu structurel. Sur l'ensemble du marché français de la publicité numérique, le duopole accumule environ $70 \%$ des investissements en 2017 et, sur le segment de la publicité sur terminaux mobiles, la concentration apparaît encore plus extrême puisque les deux acteurs catalysent 90 \% de parts de marché, marginalisant les autres acteurs de ce secteur en forte croissance ${ }^{11}$. L'automatisation et l'optimisation des achats d'espaces publicitaires se font au bénéfice des annonceurs et des intermédiaires techniques et au détriment des éditeurs et des régies.

Les acteurs des médias traditionnels apparaissent comme les grands perdants de ces mutations du marché publicitaire. Selon une étude du cabinet de conseil BearingPoint pour le ministère de la Culture et le Conseil supérieur de l'audiovisuel (CSA), l'autorité de réglementation du secteur, en France, les recettes publicitaires de la télévision, de la presse imprimée, de la radio, de l'affichage extérieur et du cinéma ont diminué d'un tiers entre 2000 et 2017. La presse apparaît particulièrement exposée à cette tendance baissière : les recettes publicitaires sur le support papier ont baissé de $71 \%$, passant de 5,4 milliards d'euros à environ 1,5 milliard d'euros sur cette même période. De plus, la transition de la presse vers le numérique n'a pas permis de canaliser les dépenses des annonceurs : l'audience globale des sites et des applications de presse a cru de près de $11 \%$ entre 2016 et 2017, en nombre total de visites. Malgré cette progression de l'audience, les recettes publicitaires numériques estimées des acteurs de la presse auraient diminué de $2 \%$ sur la même période. La télévision et la radio sont moins affectées, mais la baisse demeure soutenue : entre 2000 et 2017, les recettes publicitaires de la télévision ont baissé de $17 \%$, passant de 3,9 à 3,2 milliards d'euros, et celles de la radio, de $15 \%^{12}$.

Cette polarisation de la publicité numérique autour du duopole Google et Facebook justifie les manœuvres annoncées par des groupes de médias afin de mutualiser leurs différentes régies publicitaires en tentant de contrer cette croissance exponentielle des deux géants du secteur. En France, les groupes média français tentent de fédérer leur inventaire afin de contrer la montée en puissance de Google et de Facebook et de se réapproprier ce domaine névralgique et les profils de leurs utilisateurs. Le lancement de Gravity, qui se présente comme une alliance des médias et des data regroupant de nombreux acteurs (Centre France-La Montagne, Condé Nast, Fnac-Darty, L'Équipe, La Dépêche, La Nouvelle République, Lagardère Active, Le Télégramme, Les ÉchosLe Parisien, Marie-Claire, M6, NextRadioTV, Groupe Perdriel, Prisma Media, SFR, SoLocal et Sud-Ouest), s'inscrit dans cette logique défensive. Gravity recueille chaque mois 10 milliards de données et touche chaque jour $44 \%$ des internautes français, soit 
16 millions de personnes. Il s'agit certes d'un projet intéressant, mais celui-ci qui peine néanmoins à rivaliser avec la puissance de Google et de Facebook, qui touchent respectivement 60 et $70 \%$ des internautes en France et proposent des données très ciblées de leurs utilisateurs aux annonceurs. On notera que cette orientation vers une mutualisation des régies des médias traditionnels apparait dans d'autres pays, comme le programme EBX (European Broadcast Exchange, qui concerne cinq groupes audiovisuels de cinq pays européens, dont TF1), Nonio (qui centralise l'inventaire de 70 sites médias portugais) ou encore Verimi (regroupant les plus importants acteurs allemands).

\section{Conclusion}

Les gestionnaires de plateformes publicitaires numériques contrôlent les fonctions stratégiques en définissant les standards techniques des contenus et le niveau de rétribution des annonceurs, tout en maîtrisant la relation client. Dans cette configuration nouvelle, la production de la valeur n'est plus uniquement liée aux contenus, mais elle réside de façon croissante dans le service et les outils qui permettent de gérer l'appariement entre l'offre et la demande par des algorithmes optimisant le placement publicitaire, de manière de plus en plus efficace grâce à la collecte continue des données personnelles. Les plateformes publicitaires (Ad Exchange) apparaissent comme de nouveaux agents d'intermédiation, créant de nouvelles efficacités en agrégeant des marchés non reliés (espaces publicitaires multiples, voire invisibles, et annonceurs de toute taille), un processus par lequel les plateformes offrent des marchés centralisés pour servir des individus et des organisations très variés et dispersés. L'agrégation de marché fournit des informations efficaces pour les utilisateurs de la plateforme qui étaient auparavant engagés dans les interactions de manière aléatoire, souvent sans accès à des informations fiables ou mises à jour.

Loin de refléter la diversité du paysage publicitaire «classique » structuré autour d'une myriade de régies publicitaires et de médias (locaux, régionaux et nationaux), le marché de la publicité numérique apparaît comme un prisme sélectif largement favorable au duopole Google et Facebook. Bénéficiant de la convergence technologique, des défaillances des régulations limitant leur expansion et de ressources financières considérables, les deux acteurs ont étendu leur influence sur le marché publicitaire mondial. Leur portée dépasse largement ce domaine, menaçant plus généralement les médias souvent très dépendants des revenus publicitaires.

Ce travail sur les mutations du marché publicitaire visera ainsi, dans un second temps, à s'attarder davantage aux enjeux culturels et politiques liés à l'influence de la numérisation sur les biens culturels et informationnels. Une telle orientation permet de tenir compte des spécificités symboliques des biens médiatiques et culturels ainsi que de leurs répercussions économiques et sociales liées à la numérisation.

Les mutations et les interactions observées ont des conséquences sur la structure tarifaire, le niveau des prix (prix de vente ou tarif publicitaire) et le ratio «volume de publicité/contenu éditorial ». L'étude des secteurs des médias à partir de la théorie des marchés à deux versants met notamment en évidence la complexité des arbitrages stratégiques auxquels les éditeurs doivent faire face (Sonnac, 2013). Les récentes études (Toussaint-Desmoulins, 2015) sur la presse soulignent que les acteurs sont de moins en moins enclins à proposer du contenu gratuitement : certains journaux ont amorcé cette 
généralisation du paiement des contenus (la plupart des sites de journaux français limitent fortement l'accès aux contenus gratuits).

Soulignons que cette évolution des modèles d'affaires choisis par des éditeurs n'est pas neutre politiquement dans le domaine des médias. L'orientation des acteurs vers des modèles freemium risque d'engendrer une information à deux vitesses : le premier niveau d'accès à la plateforme serait gratuit, pour une information uniforme, consensuelle, pas chère et dépendante de la publicité, et un second niveau serait payant, in fine réservé à une certaine élite composée d'experts et de décideurs, une élite prête à payer pour une information de qualité, enrichie, premium (Sonnac, 2013). Le recul fait néanmoins défaut pour connaître l'efficacité de ces stratégies. Dans cet environnement très concurrentiel, l'évolution de la régulation dans les médias semble indispensable pour préserver l'accessibilité et la diversité de l'espace médiatique.

49 Ces dangers rejoignent les enjeux développés dans le cadre de l'économie politique de la communication, qui met notamment en question les conséquences pour l'intérêt général et la vie en société des agencements économiques de la production et de la consommation de culture et d'information (Guibert, Rebillard et Rochelandet, 2016). Ce champ théorique apparait comme un cadre conceptuel particulièrement fertile pour décrire les logiques de pouvoir qui traversent le couple médias et publicité. Cette approche s'est développée dans le domaine des media studies à l'international et dans les sciences de l'information et de la communication au niveau francophone, et est apparue dès les années 1960 aux États-Unis, notamment autour des réflexions d'Herbert Schiller sur l'hégémonie culturelle américaine et la marchandisation de l'audience à travers la publicité commerciale. Les phénomènes de concentration et de transnationalisation du marché publicitaire trouvent en effet un écho particulièrement intéressant dans ce champ de recherche. La restructuration des modes de circulation et de production des messages publicitaires ainsi que l'asymétrie du marché mise en exergue dans cet article vont-elles accroître la concentration du secteur? Les rapports de coopétition observés dans la mutualisation des régies publicitaires semblent confirmer cette tendance.

Afin de traiter plus finement l'influence de la baisse des revenus liés à la publicité numérique des médias traditionnels, il serait également intéressant d'orienter nos travaux sur le champ théorique lié aux médias et à l'espace public. La course à l'audience, notamment relative à la remise en cause du modèle économique de ces médias, engendrerait une trivialisation, une "circulation circulaire des informations » et une homogénéisation des sujets traités (Smyrnaios, 2017). La production d'informations « à cliquer » (clickbait) jouant sur des ressorts émotionnels engendre de nouveaux formats d'écriture ("Les 10 villes les plus...», « Découvrez comment...») et répand des formats d'écriture auxquels les médias traditionnels ont de plus en plus de mal à résister, notamment dans l'optique de renforcer leurs revenus publicitaires (Cardon, 2015). Il s'agirait ainsi d'interroger l'influence de ces nouvelles plateformes publicitaires sur la production et la marchandisation des contenus informationnels.

51 Sur le plan de la régulation, il conviendrait également d'étudier les mesures prises par la Commission européenne dans le cadre d'un renforcement de la protection des données personnelles : de nombreux éditeurs et plateformes publicitaires dépendent en effet des cookies, dont l'utilisation pourrait être mise à mal dans le contexte de la règlementation $e$ privacy $^{13}$. La percée de ces dispositifs d'intermédiation soulève ainsi plusieurs questions, notamment celle de la régulation de ces plateformes publicitaires, qui nécessitent une réflexion nouvelle à la lumière des changements économiques induits par la 
généralisation du modèle. Ceux-ci incluent les abus de position de dominante, les situations de concurrence déloyale, la confidentialité et la sécurisation de la collecte des données, ou encore le contrôle national des ressources d'information.

\section{BIBLIOGRAPHIE}

Allary, J. et Balusseau, V. (2018). La publicité à l'heure de la data. Paris, France : Dunod.

Brousseau, E. et Curien, N. (2001). Économie d'Internet, économie du numérique. Revue économique, 52(7), 7-36.

Bullich, V. et Guignard, T. (2011). Les plates-formes d'accès aux contenus : des dispositifs au cœur de la reconfiguration des filières communicationnelles. Actes du Colloque international Médias011 "Y'a-t-il une richesse des réseaux? ", ISIM, LID2MS, CERGAM, LSIS, Université Paul Cézanne, $145-160$.

Bouquillion, P., Miège, B. et Mœglin P. (2013). L'industrialisation des biens symboliques : les industries créatives en regard des industries culturelles. Grenoble, France : Presses universitaires de Grenoble.

Cardon, D. (2015), À quoi rêvent les algorithmes ?. Paris, France : Seuil/La République des idées.

Parker, G. G., Van Alstyne M. W. et Choudary, S. P. (2016). Platform Revolution: How Networked Markets Are Transforming the Economy - and How to Make Them Work for You. New York, NY : W. W. Norton \& Compan.

Evans, D. et Schmalensee, R. (2016), Matchmakers. Boston, MA : Harvard Business School Publishing.

Eveno, P. (2004). Médias et publicité : une association équivoque mais indispensable. Le Temps des médias, 2(1), 17-27. doi: 10.3917/tdm.002.0017

Gawer, A. (dir.), (2009). Platforms, Markets and Innovation. Londres, Angleterre : Edward Elgar Publishing.

Godin, S. (2007). Permission Marketing: Turning Strangers Into Friends And Friends Into Customers. Londres, Royaume-Uni : Simon \& Shuster.

Guibert, G., Rebillard, F. et Rochelandet, F. (2016). Médias, culture et numérique. Approches socioéconomiques. Paris, France : Armand Colin.

Hagiu, A. (2007). Merchant or Two-Sided Platform?. Review of Network Economics, 6(2), 115-133.

Lallement, G., Serris, J. et Varenne, D. (2014). L'émergence des places de marché publicitaires, l'opportunité d'une nouvelle industrie. Annales des Mines, (3), 65-69.

Ouakrat, A. (2012). Le ciblage comportemental, une perte de contrôle des éditeurs sur les données de l'audience. tic\&société, 6(1). doi: 10.4000/ ticetsociete.1251

Renaud, J..F., (2017). Les achats programmatiques : comprendre les enjeux. Gestion, (42), 106-109.

Rochet, J.-C. et Tirolle, J. (2003). Platform Competition in Two-Sided Markets. Journal of the European Economic Association, (4), 990-1029. 
Rodgers, S. et Thorson, E. (2017), Digital Advertising: Theory and Research. New York, NY : Routledge.

Rosen, S. (1981). The Economics of Superstars. The American Economic Review, 71(5), 845-858.

Smyrnaios, N. (2017). Les GAFAM contre l'internet : une économie politique du numérique. Paris, France : INA.

Sonnac, N. (2006). Médias et publicité, ou les conséquences d'une interaction entre deux marchés. Le Temps des médias, (6), 49-58.

Sonnac, N. (2013). L'écosystème des médias. Communication 32(2). doi: 10.4000/ communication.5030

Toussaint-Desmoulins, N. (2015). L'économie des médias. Paris, France : Presses universitaires de France.

\section{NOTES}

1. Les médias « traditionnels » sont les médias qui étaient établis avant Internet.

2. Dans son article marquant en 1981, "The Economics of Superstars", Sherwin Rosen, professeur à l'Université de Chicago, théorisait le concept du winner takes all. Celui-ci traduit le fait que les transformations liées à la technologie donnent un pouvoir financier immense et disproportionné à quelques acteurs déclassant les autres concurrents présents sur leur marché.

3. Pour l'ensemble de la presse, les recettes de publicité représentaient $36 \%$ des ressources contre $64 \%$ pour les ventes en 2013 (Direction générale des médias et des industries culturelles). Cette proportion a varié selon les années (47,6/52,4 en 1990, mais 40/60 en 1984) en raison des fluctuations conjoncturelles. Or, autour de cette moyenne générale, on trouve des situations très contrastées - entre divers genres de presse, divers titres d'un même genre - liées à la sélectivité de la publicité. Ainsi, tandis qu'en 2013 la presse quotidienne nationale tirait 34,8\% de ses ressources de la publicité et des petites annonces, ce pourcentage était de $34,5 \%$ pour la presse quotidienne régionale, de 48,6\% pour la presse technique et spécialisée (dont plus de 76,5\% pour la presse du bâtiment), mais de moins de $5 \%$ pour la presse des jeunes (Toussaint-Desmoulins, 2015).

4. Les chaînes du câble et du satellite, financées en 2013 en moyenne à 60 \% par l'abonnement, à $26 \%$ par des recettes diverses et à $14 \%$ par la publicité, sont pour la grande majorité d'entre elles encore déficitaires (Toussaint-Desmoulins, 2015).

5. La publicité numérique est devenue le premier média pour les annonceurs, devant la télévision, avec une valeur, en France, de plus de 4 milliards d'euros en 2017, en particulier grâce à la vidéo, aux réseaux sociaux et à la publicité " programmatique » (Bougon, F. et Piquard, A. (2018, 6 mars). Publicité numérique: l'Autorité de la concurrence appelle à davantage de transparence. Le Monde. Repéré à https://www.lemonde.fr/economie/article/2018/03/06/ publicite-numerique-l-autorite-de-la-concurrence-appelle-a-davantage-detransparence_5266355_3234.html).

6. L'achat programmatique est avant toute chose une nouvelle manière d'envisager une transaction commerciale entre un acheteur et un vendeur de publicité. Il s'agit de mettre en relation un acheteur et un vendeur en temps réel au moyen de technologies sur des places de marché qui peuvent être ouvertes ou contraintes (open ad exchange, private ad exchange). Le mode d'achat le plus courant est la mise aux enchères en temps réel (RTB) de chaque élément publicitaire d'un site ou d'une application, qu'il s'agisse de formats standards ou de vidéos (Renaud, 2017). 
7. Bayle, N. (2018, 29 janvier). Réseaux sociaux, data... la publicité à l'ère du numérique. Le Monde. Repéré à https://www.lemonde.fr/economie-mondiale/article/2018/01/29/la-publicite-tournela-page_5248526_1656941.html

8. Les investissements en recherche et en développement en 2017 sont estimés à 14 milliards de dollars US pour Alphabet (Google) et à 3,9 milliards de dollars US pour Facebook (Étude CSA, ministère de la Culture et BearingPoint, 2018).

9. BlueKai dispose d'une base de données d'environ un milliard d'utilisateurs comportant une cinquante d'attributs (Smyrnaios, 2017).

10. Dix-huitième édition de l'Observatoire de l'e-pub du SRI (Syndicat des régies Internet), réalisé par PwC en partenariat avec l'Union des entreprises de conseil et achat média (Udecam) (http:// www.sri-france.org/etudes-et-chiffre-cles/observatoire-de-le-pub-sri/18eme-observatoire-depub-sri/).

11. Ibid.

12. Ibid.

13. Ce règlement vise également à simplifier les dispositions sur les cookies en donnant plus de choix aux utilisateurs (Règlement général sur la protection des données : https://eur-lex.europa.eu/ legal-content/FR/TXT/?uri=CELEX\%3A52017PC0010

\section{RÉSUMÉS}

Les plateformes publicitaires apparaissent comme de nouveaux agents d'intermédiation créant de nouvelles efficacités en agrégeant des marchés non reliés, un processus par lequel les plateformes offrent des marchés centralisés pour servir des individus et des acteurs très dispersés. L'agrégation de marché fournit des informations efficaces pour les utilisateurs de la plateforme qui étaient auparavant engagés dans les interactions de manière aléatoire, souvent sans accès à des informations fiables ou mises à jour. S'il est vrai qu'on ne peut résumer la publicité en ligne à Google et Facebook, nous verrons que ces acteurs constituent des intermédiaires incontournables contrôlant un large pan du marché et méritent ainsi une attention particulière.

Advertising platforms are new agents of intermediation that create new efficiencies by aggregating unorganized markets, and proposing centralized markets to serve widely dispersed individuals and organizations. Market aggregation provides effective information for platform users who were previously engaged in random interactions, often without access to reliable or up-to-date information. Although online advertising cannot be reduced to Google and Facebook, these players are nonetheless essential intermediaries controlling a large part of the market and thus deserve special attention.

Las plataformas de publicidad aparecen como nuevos agentes de intermediación que crean nuevas eficiencias al agregar mercados no organizados, un proceso por el cual las plataformas ofrecen mercados centralizados para servir a individuos y organizaciones ampliamente dispersos. La agregación de mercados proporciona información efectiva para los usuarios de la plataforma que anteriormente participaban en interacciones aleatorias, a menudo sin acceso a información fiable o actualizada. Si bien es cierto que la publicidad online no se puede resumir en 
Google y Facebook, veremos que estos actores son intermediarios esenciales que controlan gran parte del mercado y por lo tanto merecen una atención especial.

\section{INDEX}

Mots-clés : médias, publicité, plateforme, numérique, données personnelles, appariement Palabras claves : media, publicidad, plataforma, información personal, coincidencia Keywords : media, advertising, platform, digital, personal data, matching

\section{AUTEUR}

\section{THOMAS GUIGNARD}

Thomas Guignard est rattaché au laboratoire GERIICO depuis septembre 2017. Ses recherches actuelles portent essentiellement sur les traces et les plateformes numériques. L'intégration du couple « dispositif algorithmique/Big Data » au sein des plateformes numériques constitue une composante de ses recherches en cours. Il s'intéresse aux processus d'industrialisation, de technicisation et de marchandisation des biens culturels et informationnels.

thomas.guignard@univ-lille.fr 